\title{
PKB/Akt phosphorylation of ERR $\gamma$ contributes to insulin-mediated inhibition of hepatic gluconeogenesis
}

\author{
Don-Kyu Kim • Yong-Hoon Kim • Debby Hynx • Yanning Wang • Keum-Jin Yang • \\ Dongryeol Ryu • Kyung Seok Kim • Eun-Kyung Yoo • Jeong-Sun Kim • Seung-Hoi Koo • \\ In-Kyu Lee • Ho-Zoon Chae • Jongsun Park • Chul-Ho Lee • Sudha B. Biddinger • \\ Brian A. Hemmings $\cdot$ Hueng-Sik Choi
}

Received: 5 June 2014 / Accepted: 6 August 2014 / Published online: 10 September 2014

(C) Springer-Verlag Berlin Heidelberg 2014

\begin{abstract}
Aims/hypothesis Insulin resistance, a major contributor to the pathogenesis of type 2 diabetes, leads to increased hepatic glucose production (HGP) owing to an impaired ability of insulin to suppress hepatic gluconeogenesis. Nuclear receptor oestrogen-related receptor $\gamma(\mathrm{ERR} \gamma)$ is a major transcriptional regulator of hepatic gluconeogenesis. In this study, we investigated insulin-dependent post-translational modifications (PTMs) altering the transcriptional activity of ERR $\gamma$ for the regulation of hepatic gluconeogenesis.

Methods We examined insulin-dependent phosphorylation and subcellular localisation of ERR $\gamma$ in cultured cells and in
\end{abstract}

Electronic supplementary material The online version of this article (doi:10.1007/s00125-014-3366-x) contains peer-reviewed but unedited supplementary material, which is available to authorised users.

D.-K. Kim $\cdot$ H.-S. Choi $(\bowtie)$

National Creative Research Initiatives Center for Nuclear Receptor

Signals and Hormone Research Center, School of Biological

Sciences and Technology, Chonnam National University,

Gwangju 500-757, Republic of Korea

e-mail: hsc@chonnam.ac.kr

Y.-H. Kim • C.-H. Lee

Korea Research Institute of Bioscience and Biotechnology, Daejeon, Republic of Korea

D. Hynx $\cdot$ B. A. Hemmings

The Friedrich Miescher Institute for Biomedical Research, Basel, Switzerland

Y. Wang $\cdot$ S. B. Biddinger

Division of Endocrinology, Children's Hospital Boston, Harvard

Medical School, Boston, USA

K.-J. Yang $\cdot$ J. Park

Department of Pharmacology, Daejeon Regional Cancer Center, Cancer Research Institute, Chungnam National University, Taejeon, Republic of Korea the liver of C57/BL6, leptin receptor-deficient ( $d b / d b)$, liverspecific insulin receptor knockout $($ LIRKO) and protein kinase $\mathrm{B}$ (PKB) $\beta$-deficient $\left(P k b \beta^{--}\right)$mice. To demonstrate the role of ERR $\gamma$ in the inhibitory action of insulin on hepatic gluconeogenesis, we carried out an insulin tolerance test in C57/BL6 mice expressing wild-type or phosphorylationdeficient mutant ERR $\gamma$.

Results We demonstrated that insulin suppressed the transcriptional activity of ERR $\gamma$ by promoting PKB/Aktmediated phosphorylation of ERR $\gamma$ at S179 and by eliciting translocation of ERR $\gamma$ from the nucleus to the cytoplasm through interaction with 14-3-3, impairing its ability to

\section{Ryu $\cdot$ S.-H. Koo}

Division of Life Sciences, College of Life Sciences and

Biotechnology, Korea University, Seoul 136-701, Republic of Korea

K. S. Kim • H.-Z. Chae

School of Biological Sciences and Technology, Chonnam National University, Gwangju, Republic of Korea

E.-K. Yoo - I.-K. Lee

Department of Internal Medicine, Kyungpook National University School of Medicine, Deagu, Republic of Korea

J.-S. Kim

Department of Chemistry and Institute of Basic Sciences, Chonnam National University, Gwangju, Republic of Korea 
promote hepatic gluconeogenesis. In addition, $d b / d b$, LIRKO and $P k b \beta^{-1-}$ mice displayed enhanced ERR $\gamma$ transcriptional activity due to a block in PKB $\beta$-mediated ERR $\gamma$ phosphorylation during refeeding. Finally, the phosphorylation-deficient mutant ERR $\gamma$ S179A was resistant to the inhibitory action of insulin on HGP.

Conclusions/interpretation These results suggest that ERR $\gamma$ is a major contributor to insulin action in maintaining hepatic glucose homeostasis.

Keywords Akt/PKB · Hepatic gluconeogenesis · Insulin receptor signalling $\cdot$ Nuclear hormone receptor .

Phosphorylation

$\begin{array}{ll}\text { Abbreviations } \\ \text { Ad } & \text { Adenovirus } \\ \text { cAMP } & \text { Cyclic AMP } \\ \text { ChIP } & \text { Chromatin immunoprecipitation } \\ \text { DBD } & \text { DNA-binding domain } \\ \text { dsDNA } & \text { Double-stranded DNA } \\ \text { ERR } & \text { Oestrogen-related receptor } \\ \text { ERR } \gamma \text { wt } & \text { Wild-type ERR } \gamma \\ \text { ERRE } & \text { ERR response element } \\ \text { FOXO1 } & \text { Forkhead box O1 } \\ \text { GST } & \text { Glutathione } S \text {-transferase } \\ \text { HGP } & \text { Hepatic glucose production } \\ \text { ICC } & \text { Immunocytochemistry } \\ \text { IHC } & \text { Immunohistochemistry } \\ \text { IR } \beta & \text { Insulin receptor } \beta \\ \text { LIRKO } & \text { Liver-specific insulin receptor knockout } \\ \text { PGC-1 } \alpha & \text { Peroxisome proliferator-activated } \\ & \text { receptor } \gamma \text { coactivator 1- } \alpha \\ \text { PI3 } & \text { Phosphatidylinositol } 3 \\ \text { PTM } & \text { Post-translational modification } \\ \text { PKB } & \text { Protein kinase B } \\ \text { PKB ca } & \text { Constitutively active PKB } \\ \text { SHP } & \text { Small heterodimer partner } \\ \text { WT } & \text { Wild-type } \\ & \end{array}$

\section{Introduction}

Hepatic glucose metabolism is controlled primarily by the actions of counter-regulatory hormones: glucagon, acting through cyclic AMP (cAMP)-dependent pathways, and insulin, acting through the phosphatidylinositol 3 (PI3)-kinase pathway [1]. During fasting, glucagon contributes to the maintenance of constant levels of plasma glucose through breakdown of glycogen stored in liver via glycogenolysis and through de novo synthesis (gluconeogenesis) of additional glucose from lactate, pyruvate, glycerol and amino acids [2]. Regulation of hepatic gluconeogenesis is associated with a number of transcription factors that contribute to the transcriptional regulation of rate-limiting key gluconeogenic enzyme genes such as phosphoenolpyruvate carboxykinase (Pckl) and glucose-6-phosphatase (G6pc) [3]. During feeding, insulin mainly inhibits hepatic glucose production (HGP) through inactivation of the transcription factor, forkhead box O1 (FOXO1), by protein kinase B (PKB)-mediated phosphorylation. However, additional transcriptional regulators, including cAMP response element-binding protein (CREB)regulated transcription coactivator 2 , peroxisome proliferator-activated receptor $\gamma$ coactivator $1-\alpha$ (PGC- $1 \alpha)$ and FOXO6, have also been reported to mediate insulin inhibition of gluconeogenesis in liver; and abnormal regulation of these factors is associated with hepatic insulin resistance [4-6].

The oestrogen-related receptor (ERR) subfamily of nuclear receptors consists of three members, ERR $\alpha, E R R \beta$ and ERR $\gamma$ (NR3B1-3), which bind to classic oestrogen response elements as dimers, or to extended half-site core sequences as monomers [7]. The amino acid sequences of the three ERR isoforms are highly similar in the DNA-binding domain (DBD). In addition, the transcriptional activity of ERRs that are constitutively active in the absence of endogenous ligand depends mainly on interaction with coactivator or corepressor proteins. It is reported that the transcriptional activity of ERR $\gamma$ plays an important role in the regulation of glucose, lipid, alcohol and iron metabolism in mouse liver $[8,9]$. Furthermore, hepatic ERR $\gamma$ expression is induced in fasting or diabetic conditions, and the increased transcriptional activity of ERR $\gamma$ causes insulin resistance and glucose intolerance through induction of hepatic gluconeogenesis [10,11]. In addition, induction of ERR $\gamma$ in liver leads to impaired insulin signalling through diacylglycerol-mediated protein kinase $\varepsilon$ activation [12], suggesting that ERR $\gamma$ transcriptional activity could be involved in insulin action to maintain glucose homeostasis. In the current study, we investigated insulin-dependent post-translational modification (PTM) altering the transcriptional activity of $\mathrm{ERR} \gamma$ in the regulation of hepatic gluconeogenesis.

\section{Methods}

Animal experiments Animal experiments were performed using 8-week-old male C57BL/6J, $d b / d b$, liver-specific insulin receptor knockout (LIRKO) and $P k b \beta^{-1-}$ (also known as $A k t 2^{-1-}$ ) mice. C57BL/6J and $d b / d b$ mice were obtained from Jackson Laboratories (Bar Harbor, ME, USA) and liver and serum of LIRKO and $P k b \beta^{-1-}$ mice were provided by Dr. S. B. Biddinger (Harvard Medical School, Boston, MA, USA) and Dr. B. A. Hemmings (Friedrich Miescher Institute for Biomedical Research, Basel, Switzerland) as described previously $[13,14]$. All mice were acclimatised to a $12-\mathrm{h}$ light/dark cycle at $22 \pm 2{ }^{\circ} \mathrm{C}$ for 2 weeks with free access to food and water in a specific pathogen-free facility. To identify 
the effect of insulin on ERR $\gamma$-mediated induction of hepatic gluconeogenesis in vivo, recombinant adenoviruses (Ad-GFP, Ad-Flag-ERR $\gamma$ and Ad-Flag-S179A; $1 \times 10^{9}$ plaque-forming units) were delivered by tail-vein injection into 8 -week-old male C57BL/6J mice. Plasma glucose levels were determined with an automated blood chemistry analyser (Hitachi 7150; Tokyo, Japan) and plasma insulin concentrations were measured using a mouse insulin ELISA kit (ALPCO Diagnostics, Salem, NH, USA). All animal experiments were approved and performed by the Institutional Animal Use and Care Committee of the Chonnam National University.

Analytical methods Chemicals and DNA constructions are described in the electronic supplementary material (ESM) Methods. Rabbit anti-phospho-ERR $\gamma$ was generated as described previously [15]. See ESM Methods for further details.
The in vitro kinase assay was performed as described previously [16]. See ESM Methods for further details. For western blot analysis, whole-cell extracts were prepared using radioimmunoprecipitation assay (RIPA) buffer (ElpisBiotech, Daejeon, Korea) with protease inhibitor cocktail set III (Calbiochem, San Diego, CA, USA) and phosphatase inhibitor cocktail set III (Calbiochem). See ESM Methods for further details. The glucose output assay was performed as described previously [10]. The chromatin immunoprecipitation (ChIP) assay was performed according to the manufacturer's protocol (Millipore, Billerica, MA, USA). See ESM Methods for further details. Primary hepatocytes were isolated from Sprague-Dawley rats (male, 180-300 g, Samtako BioKorea, Seoul, Korea) by collagenase perfusion, as described previously [10]. For transient transfection assays, HepG2 (human hepatoblastoma-derived cells), AML12 a

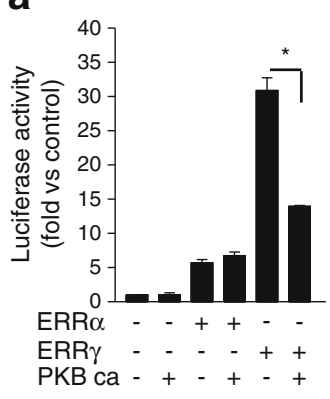

d

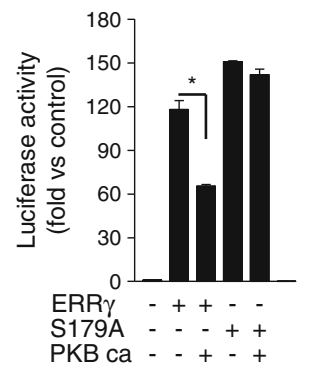

e

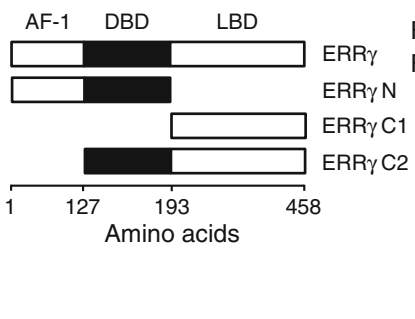

g

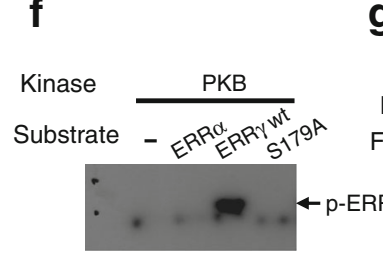

b

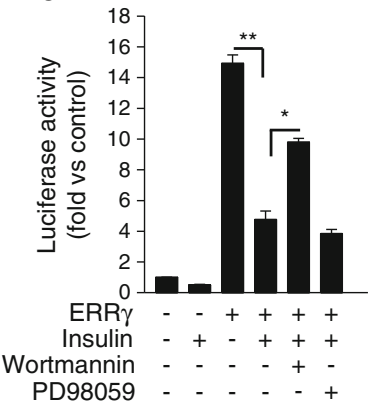

C

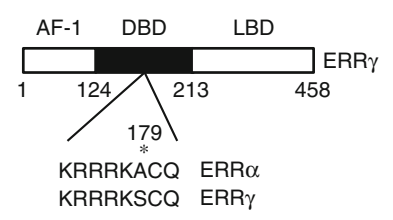

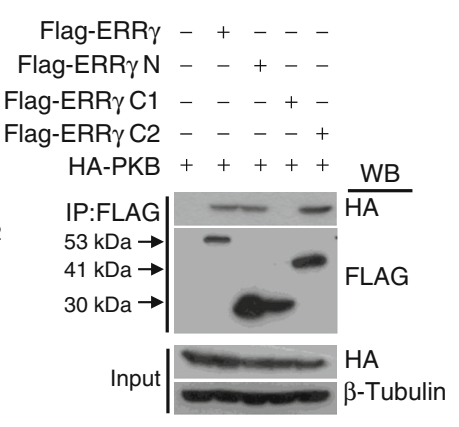

h

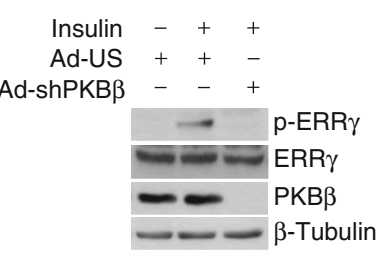

Fig. 1 Insulin inhibits ERR $\gamma$ activity through PKB-mediated phosphorylation at S179. (a) PKB effect on ERR $\gamma$ transactivity in 293T cells (shown as luciferase activity, sft4-luc) $(+, 200 \mathrm{ng}) ;{ }^{*} p<0.05$ vs ERR $\gamma$. (b) Effect of insulin on ERR $\gamma$ transactivity in 293T cells $(+, 100 \mathrm{ng})$ exposed to wortmannin $(100 \mathrm{nmol} / \mathrm{l})$ or PD98059 $(10 \mu \mathrm{mol} / \mathrm{l})$ for $24 \mathrm{~h}$ prior to $18 \mathrm{~h}$ treatment with insulin $(100 \mathrm{nmol} / \mathrm{l}) ;{ }^{*} p<0.05$ and ${ }^{* *} p<0.01$ as shown. (c) Alignment of amino acid residues flanking a putative phosphorylation site of ERR $\alpha$ and ERR $\gamma$. The asterisk shows a substitution. (d) PKB effect on ERR $\gamma$ S179A transactivity in 293T cells (+,
$300 \mathrm{ng}) ;{ }^{*} p<0.05$ vs ERR $\gamma$. (e) Domain mapping of ERR $\gamma$ interacting with PKB in 293T cells. The cell lysates were immunoprecipitated with anti-FLAG. (f) In vitro PKB kinase assay. (g) Transient transfection assay showing insulin-mediated ERR $\gamma$ phosphorylation in $293 \mathrm{~T}$ cells. Insulin $(100 \mathrm{nmol} / \mathrm{l})$ was added for $18 \mathrm{~h}$. (h) Effect of PKB $\beta$ knockdown on ERR $\gamma$ phosphorylation in AML12 cells treated with insulin $(100 \mathrm{nmol} / \mathrm{l})$ for $18 \mathrm{~h}$. Data are presented as means \pm SEM. The experiment was repeated on a minimum of three separate occasions 
(alpha mouse liver 12, a non-transformed mouse liver cell line) and $293 \mathrm{~T}$ cells were maintained as described previously [10]. See ESM Methods for further details. Immunofluorescence staining was performed according to the manufacturer's protocol for Alexa Fluor SFX kits (Molecular Probes, Invitrogen, Carlsbad, CA USA). See ESM Methods for further details. Quantitative PCR was performed as described previously [10]. See ESM Methods for further details.

Statistical analyses All values are expressed as means \pm SEM. The significance between mean values was evaluated by two-tailed unpaired Student's $t$ test.

\section{Results}

Insulin inhibits ERR $\gamma$ transcriptional activity via PKB-mediated phosphorylation at $S 179$ Since ERR $\alpha$ and ERR $\gamma$ are associated with hepatic glucose metabolism, we first tested if insulin affects the transcriptional activity of $\mathrm{ERR} \alpha$ and ERR $\gamma$ on reporter construct sft4-luc containing three copies of ERR-binding sites (TCAAGGTTG). This reporter is driven by the promoter of the gene encoding orphan nuclear receptor small heterodimer partner (SHP), which is a known target of both ERR $\alpha$ and ERR $\gamma$ [17]. Interestingly, transcriptional activity of ERR $\gamma$, but not of ERR $\alpha$, on sft4-luc was significantly decreased by cotransfection of constitutively active PKB (PKB ca) into 293 T cells (Fig. 1a). In addition, treatment with wortmannin, a PI3-kinase inhibitor, significantly restored the insulin-mediated inhibition of ERR $\gamma$ transcriptional activity, whereas treatment with PD98059, a mitogen-activated protein kinase inhibitor, did not significantly change the inhibitory effect of insulin on ERR $\gamma$ (Fig. 1b). These results raised the possibility that PKB could modulate the transcriptional activity of ERR $\gamma$ in response to insulin. Consistent with this idea, the primary structure of ERR $\gamma$ revealed the presence of a potential $\mathrm{PKB}$ phosphorylation site in a DBD domain of ERR $\gamma$ (Fig. 1c), which was highly conserved among species from fish to mammals (ESM Fig. 1a). However, no PKB phosphorylation site was found in ERR $\alpha$ (Fig. 1c). Next, to test if the site is involved in PKB-mediated phosphorylation, we generated ERR $\gamma$ S179A (a Ser-to-Ala substitution), a phosphorylation-deficient mutant, and ERR $\gamma$ S179D (a Ser-to-Asp substitution), a phospho-mimic mutant, and found that their levels were comparable with that of wild-type (WT) ERR $\gamma$ (ERR $\gamma$ wt), as judged by western blot analysis (ESM Fig. 1b). As expected, basal transcriptional activity of ERR $\gamma$ S179D was markedly decreased compared with that of ERR $\gamma$ wt, whereas basal ERR $\gamma$ S179A activity was considerably higher than that of ERR $\gamma$ wt and was unaffected by PKB ca (Fig. 1d and ESM Fig. 1c). These results suggest that S179 of ERR $\gamma$ plays an important role in the insulin-mediated regulation of ERR $\gamma$ transcriptional activity.

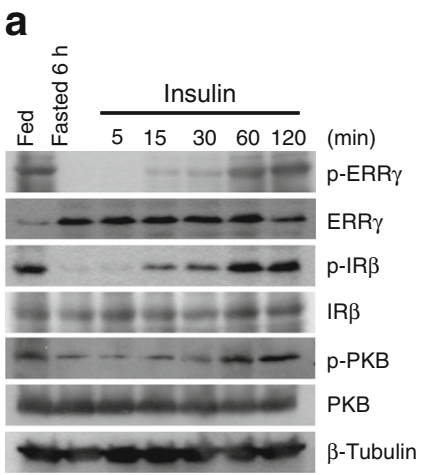

d

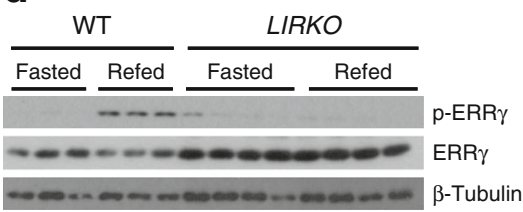

Fig. 2 ERR $\gamma$ is phosphorylated through insulin receptor signalling during refeeding. (a) Western blot analysis showing phospho- and total ERR $\gamma, \operatorname{IR} \beta$ and PKB protein levels in liver. C57/BL6 mice $(n=3$ per group) were fed ad libitum, fasted for $6 \mathrm{~h}$, or fasted for $6 \mathrm{~h}$ and then injected with insulin $(1 \mathrm{U} / \mathrm{kg})$. (b) Western blot analysis showing phospho- and total ERR $\gamma$, IRS-1 and PKB protein levels in liver of ad libitum fed, fasted and refed C57/BL6 mice ( $n=3$ per group). (c) Western blot analysis showing phospho- and total ERR $\gamma$, IRS-1 and PKB protein b

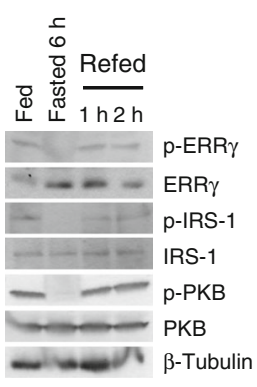

C

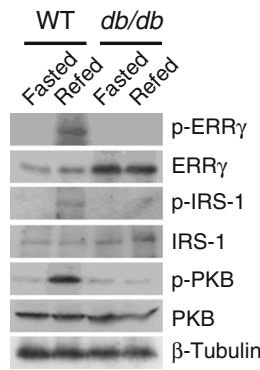

e

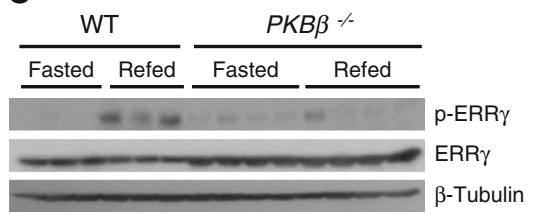

levels in liver of WT $(n=3)$ and $d b / d b(n=4)$ mice fasted for $6 \mathrm{~h}$, or fasted for $6 \mathrm{~h}$ and then refed for $2 \mathrm{~h}$. (d) Western blot analysis showing phosphoand total ERR $\gamma$ protein levels in liver of WT and LIRKO mice fasted for $6 \mathrm{~h}$, or fasted for $6 \mathrm{~h}$ and then refed for $2 \mathrm{~h}$ ( $n=4$ per group). (e) Western blot analysis showing phospho- and total ERR $\gamma$ protein levels in liver of WT $(n=4)$ and $P K B \beta^{-1-}(n=7)$ mice fasted for $6 \mathrm{~h}$, or fasted for $6 \mathrm{~h}$ and then refed for $2 \mathrm{~h}$ 
Next, we examined the interaction between ERR $\gamma$ and PKB in 293T cells, and found that ERR $\gamma$, but not ERR $\alpha$, strongly interacted with PKB through the DBD domain containing the putative phosphorylation site (Fig. 1e and ESM Fig. 1d). Moreover, an in vitro kinase assay with $\gamma-\left[{ }^{32} \mathrm{P}\right]$ showed that active PKB directly phosphorylated ERR $\gamma$ wt, but not ERR $\gamma$ S179A and ERR $\alpha$ (Fig. 1f), indicating that $\mathrm{ERR} \gamma$ is a direct substrate of PKB. To further demonstrate PKB-mediated phosphorylation of ERR $\gamma$, we generated a phospho-specific antibody that can detect the phosphorylated form at ERR $\gamma$ S179, and examined insulin-mediated phosphorylation of ERR $\gamma$ in 293T cells. As expected, insulin increased ERR $\gamma$ phosphorylation, but not ERR $\gamma$ S179A (Fig. 1g). In addition, the ERR $\gamma$ phosphorylation by insulin was induced in a time-dependent manner (ESM Fig. 1e). We also examined the effect of an ERR $\gamma$-specific inverse agonist, GSK5182, on insulin-dependent phosphorylation of ERR $\gamma$ in $293 \mathrm{~T}$ cells, and found that GSK5182 did not significantly affect the ERR $\gamma$ phosphorylation levels upon insulin treatment (ESM Fig. 1f). Finally, knockdown of PKB $\beta$ expression, a major PKB isoform in liver, almost completely abolished the insulin-mediated ERR $\gamma$ phosphorylation (Fig. 1h).
We conclude that ERR $\gamma$ transcriptional activity is inhibited by PKB phosphorylation at S179 in response to insulin.

$E R R \gamma$ phosphorylation is involved in insulin receptor signalling during refeeding To examine the effect of insulin on ERR $\gamma$ phosphorylation in vivo, we performed intraperitoneal injections of insulin into C57/BL6 mice fasted for $6 \mathrm{~h}$. Hepatic ERR $\gamma$ phosphorylation after the insulin injection was gradually increased until $2 \mathrm{~h}$, which was consistent with the activation of insulin receptor $\beta$ (IR $\beta$ ) and PKB, downstream effectors of insulin (Fig. 2a), while blood glucose levels were gradually reduced after the insulin injection (ESM Fig. 2a). Since insulin is a major pancreatic hormone regulating hepatic glucose metabolism during the fed state, we next investigated whether hepatic ERR $\gamma$ phosphorylation is induced by refeeding. Indeed, refeeding after $6 \mathrm{~h}$ of fasting led to marked induction of ERR $\gamma$ phosphorylation and activation of IR $\beta$, IRS-1 and PKB inhibiting hepatic gluconeogenesis in mice (Fig. 2b). On the other hand, $d b / d b$ mice, an insulin-resistant diabetic model, did not display elevated phosphorylation of ERR $\gamma$ and activation of insulin receptor signalling in liver during refeeding (Fig. 2c), resulting in increased blood a

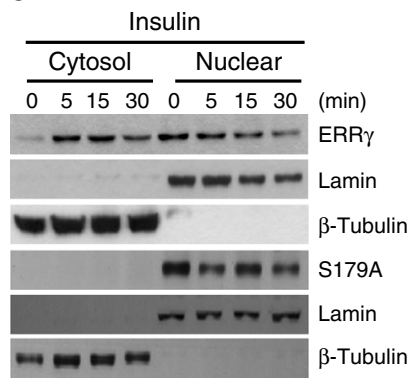

C

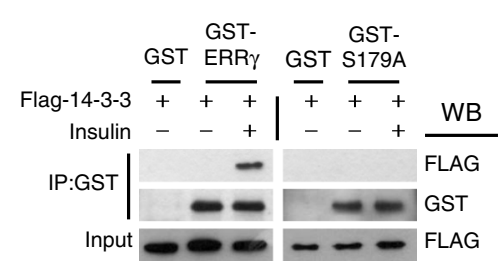

b

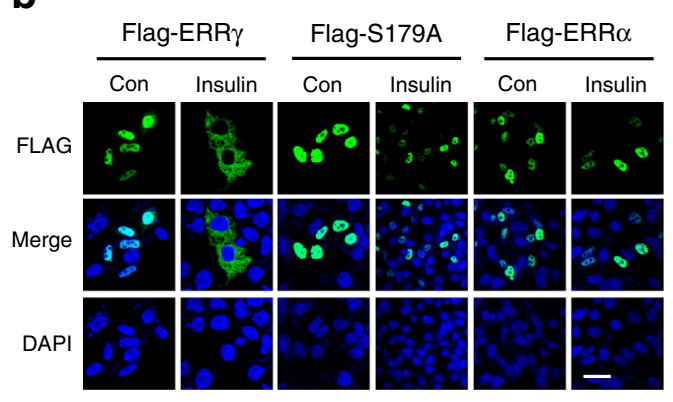

d

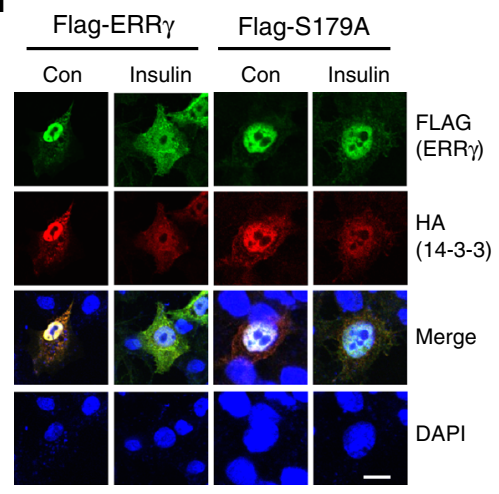

14-3-3 in 293T cells. The cells were transfected with plasmids expressing GST only, GST-ERR $\gamma$, GST-ERR $\gamma$ S179A, or Flag-14-3-3, and then treated with insulin for $18 \mathrm{~h}$. (d) ICC showing subcellular localisation of ERR $\gamma$, ERR $\gamma$ S179A and 14-3-3 in HepG2 cells. The cells were transfected with plasmids expressing HA-14-3-3 and Flag-ERR $\gamma$ or Flag-ERR $\gamma$ S179A, and then treated with insulin for $6 \mathrm{~h}$. Scale bar, $10 \mu \mathrm{m}$. The experiment was repeated on a minimum of three separate occasions 
glucose levels and hepatic gluconeogenic gene expression (ESM Fig. 2b, c). To further demonstrate insulin-mediated ERR $\gamma$ phosphorylation in vivo, we employed LIRKO and $P k b \beta^{-1-}$ mice. Consistent with the results from $d b / d b$ mice, enhanced ERR $\gamma$ phosphorylation shown during refeeding in WT mice was abolished in both LIRKO and $P k b \beta^{-/-}$mice (Fig. 2d, e). LIRKO and $P k b \beta^{-1-}$ mice exhibited hyperinsulinaemia, hyperglycaemia and elevated hepatic gluconeogenic gene expression during refeeding compared with WT mice (ESM Fig. 3). These results suggest that insulinmediated ERR $\gamma$ phosphorylation is operative in vivo.

Insulin-mediated ERR $\gamma$ phosphorylation leads to its nuclear export To explore the mechanisms underlying the inhibitory effect of insulin on the transcriptional activity of ERR $\gamma$, we examined whether insulin-mediated ERR $\gamma$ phosphorylation leads to a change in subcellular localisation. Surprisingly, ERR $\gamma$ wt, which was mainly localised to the nucleus without insulin stimulation, was rapidly translocated to the cytoplasm of 293 T cells upon insulin treatment (Fig. 3a). However, this translocation was not observed for ERR $\gamma \mathrm{S} 179 \mathrm{~A}$. To verify the cell fractionation findings, we conducted immunocytochemistry (ICC) in HepG2 cells transfected with ERR $\gamma$ wt, ERR $\gamma \mathrm{S} 179 \mathrm{~A}$ and $\mathrm{ERR} \alpha$, and found that all of them were exclusively localised in the nucleus before insulin stimulation (Fig. 3b). However, only ERR $\gamma$ wt was translocated to the cytoplasm after exposure to insulin, recapitulating the cell fractionation findings. To investigate the mechanism regarding the insulin-dependent subcellular redistribution of ERR $\gamma$, we examined the association of ERR $\gamma$ with 14-3-3, a phosphorylation-dependent scaffold protein involved in subcellular redistribution [18]. A co-immunoprecipitation study performed in 293T cells indicated that 14-3-3 strongly interacted with glutathione $S$-transferase (GST)-conjugated ERR $\gamma$ wt in the presence of insulin, but not with GST itself or ERR $\gamma$ S179A (Fig. 3c). Consistent with these results, an ICC study performed in HepG2 cells demonstrated that ERR $\gamma$ wt was co-localised with 14-3-3 in the cytoplasm after insulin stimulation, which was not observed for ERR $\gamma$ S179A (Fig. 3d). In addition, a molecular docking model of ERR $\gamma$-DBD to double-stranded DNA (dsDNA) showed that S179 of ERR $\gamma$ was located near the phosphate moieties of the bound dsDNA, causing a charge repulsion between the phospho-ERR $\gamma \mathrm{S} 179$ and the phosphate moieties of dsDNA, thereby dissociating ERR $\gamma$ from dsDNA (ESM Fig. 4). Furthermore, a monomeric ERR $\gamma$-ligand binding domain can be located within the dimeric 14-3-3 cleft while maintaining similar interactions between the phospho-ERR $\gamma$ S179 and a

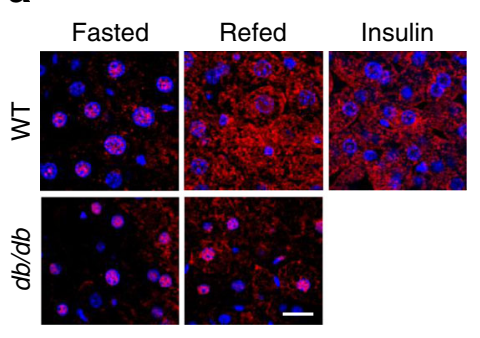

C

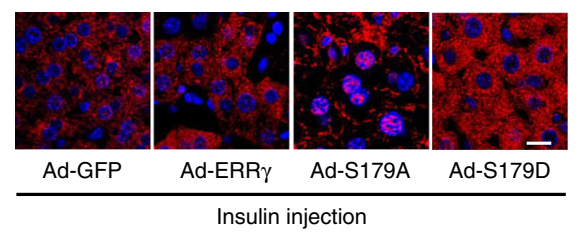

b

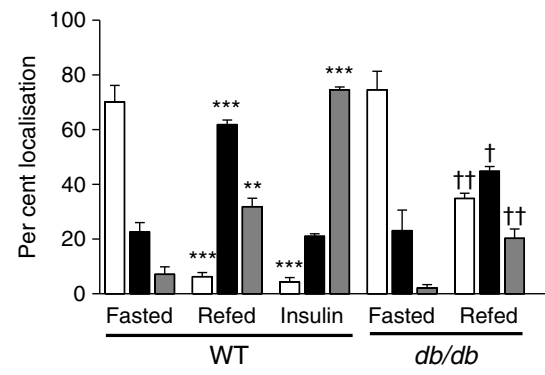

d

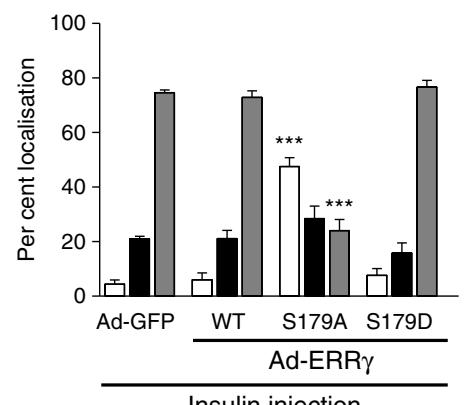

Insulin injection

( $n=5$ per group) were infected with Ad-GFP, Ad-Flag-ERR $\gamma$, Ad-FlagERR $\gamma$ S179A or Ad-Flag-ERR $\gamma$ S179D and intraperitoneally administered with insulin $(1 \mathrm{U} / \mathrm{kg}$ ) for $1 \mathrm{~h}$ after $4 \mathrm{~h}$ fasting at day 7 of adenoviral infections. IHC was performed using anti-ERR $\gamma$ antibody. Scale bar, $20 \mu \mathrm{m}$. (d) Quantification of subcellular localisation of ERR $\gamma$. $* * * p<0.001$ vs WT. White bars, nucleus; black bars, both; grey bars, cytosol. Data are presented as means \pm SEM
Fig. 4 Subcellular localisation of ERR $\gamma$ by insulin in mice. (a) Subcellular localisation of ERR $\gamma$ in the mouse liver. IHC was performed in the liver of WT $(n=3)$ and $d b / d b(n=4)$ mice fasted for $12 \mathrm{~h}$, fasted for $6 \mathrm{~h}$ and then refed for $2 \mathrm{~h}$, and fasted for $6 \mathrm{~h}$ and then injected with insulin $(1 \mathrm{U} / \mathrm{kg}$ ) for $2 \mathrm{~h}$. IHC was performed using anti-ERR $\gamma$ antibody. Scale bar, $20 \mu \mathrm{m}$. (b) Quantification of subcellular localisation of ERR $\gamma$; ${ }^{* *} p<0.01, * * * p<0.001$ vs WT-fasted. ${ }^{\dagger} p<0.05,{ }^{\dagger} p<0.01$ vs $d b / d b-$ fasted. (c) Subcellular localisation of ERR $\gamma$ in the liver. C57/BL6 mice 
bovine 14-3-3 (ESM Fig. 4). These results suggest that insulin-mediated ERR $\gamma$ phosphorylation elicits its cytoplasmic translocation through interaction with 14-3-3.

Consistent with the results in the cultured cell line, in vivo subcellular localisation analysis using immunohistochemistry (IHC) in the liver of WT mice demonstrated that ERR $\gamma$, which was exclusively localised in the nucleus during fasting, was translocated to the cytoplasm by refeeding and insulin injection (Fig. $4 \mathrm{a}, \mathrm{b}$ ). On the other hand, in $d b / d b$ mice, it was mainly distributed in the nucleus during both fasting and refeeding. In addition, the nuclear export of ERR $\gamma$ by insulin stimulation was exclusively detected in the liver of Ad-ERR $\gamma$ wt mice, whereas insulin did not affect the nuclear localisation of ERR $\gamma$ S179A (Fig. 4c, d). The phospho-mimic mutant ERR $\gamma$ S179D showed cytoplasmic localisation. These results indicate that insulin-mediated ERR $\gamma$ phosphorylation leads to its nuclear export.

Insulin inhibits hepatic gluconeogenesis through inactivation of ERR $\gamma$ On the basis of the reports that ERR $\gamma$ is a transcriptional regulator of hepatic gluconeogenesis $[10,11]$, we examined the effect of insulin on ERR $\gamma$-mediated induction of hepatic gluconeogenic gene expression. ERR $\gamma$-induced Pckl promoter activity was significantly inhibited by co-transfection with PKB ca into $293 \mathrm{~T}$ cells, while the phosphorylation-deficient mutant ERR $\gamma$ S179A was resistant to PKB-mediated inhibition of Pckl promoter activity (Fig. 5a). Consistent with the results in cultured cells, the infection of rat primary hepatocytes with Ad-ERR $\gamma$ wt led to drastic induction of Pckl, G6pc and pyruvate dehydrogenase kinase, isoenzyme 4 ( $P d k 4)$ gene expressions compared with infection with Ad-GFP, which was significantly reduced by insulin treatment (Fig. 5b). However, insulin failed to inhibit those gene expressions induced by mutant Ad-ERR $\gamma$ S179A. Interestingly, GSK5182 significantly inhibited both ERR $\gamma$ wt and ERR $\gamma$ S179A-induced Pck1 gene expression in AML12 cells (ESM Fig. 5). This suggests that the ERR $\gamma$ inverse agonist could provide an approach to suppress the transcriptional activity of ERR $\gamma$ S179A resistant to the inhibitory effect of insulin on hepatic gluconeogenesis. In addition, ChIP assays performed in rat primary hepatocytes showed that insulin disrupted the association of ERR $\gamma$ wt, but not of ERR $\gamma$ S179A with ERR response element (ERRE) 1 and -2 of the Pck1 promoter (Fig. 5c). Furthermore, glucose output assays carried out in rat primary hepatocytes showed that insulin treatment significantly attenuated glucose output induced by forskolin or Ad-ERR $\gamma$ wt, but not by Ad-ERR $\gamma$ S179A (Fig. 5d). These results suggest that the inhibitory effect of insulin on hepatic gluconeogenesis depends on ERR $\gamma$ inactivation through phosphorylation at S179.

On the strength of these results, we next tested whether the mutant ERR $\gamma$ S179A is resistant to the inhibitory action of insulin on hepatic gluconeogenesis in vivo. As expected,

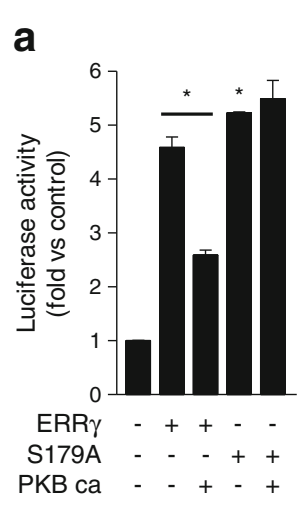

b
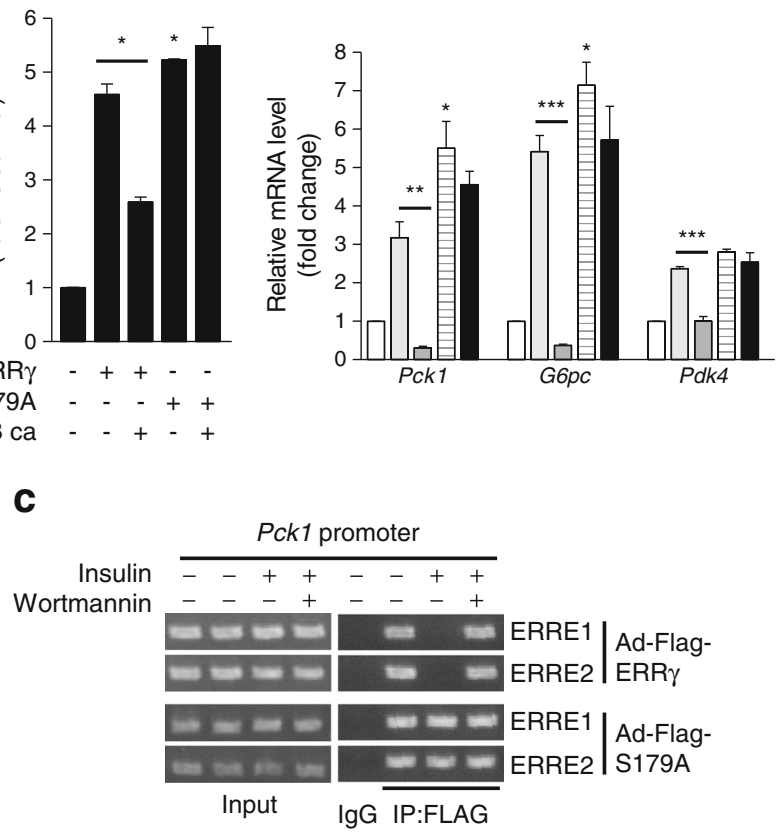

d

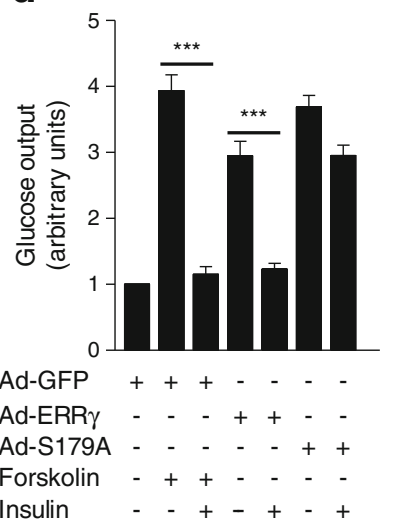

Fig. 5 ERR $\gamma$ S179A is resistant to inhibition of hepatic gluconeogenesis by insulin. (a) The inhibitory effect of PKB on ERR $\gamma$ wt or ERR $\gamma$ S179A-mediated $P c k 1$ promoter activity (as luciferase activity, Pck1-luc) in 293 T cells transfected with the indicated plasmids; ${ }^{*} p<0.05$ vs ERR $\gamma$. (b) Effect of PKB on ERR $\gamma$-mediated gluconeogenic gene expression in rat primary hepatocytes. Insulin $(100 \mathrm{nmol} / \mathrm{l})$ was added for $24 \mathrm{~h}$. White bars, Ad-GFP; light grey bars, Ad-ERR $\gamma$; dark grey bars, Ad-ERR $\gamma+$ insulin; black striped bar, Ad-S179A; black bars, Ad-S179A + insulin. ${ }^{*} p<0.05,{ }^{* *} p<0.01,{ }^{* * *} p<0.001$ vs Ad-ERR $\gamma$. (c) ChIP assay showing occupancy of ERR $\gamma$ and ERR $\gamma$ S179A on Pckl promoter. Rat primary hepatocytes were infected with Ad-Flag-ERR $\gamma$ and Ad-Flag-ERR $\gamma$ S179A and treated with wortmannin $(100 \mathrm{nmol} / \mathrm{l})$ for $24 \mathrm{~h}$ or insulin $(100 \mathrm{nmol} / \mathrm{l})$ for $18 \mathrm{~h}$. Soluble chromatin was immunoprecipitated with anti-FLAG antibody. (d) Glucose output assay. Rat primary hepatocytes were infected with Ad-GFP, Ad-ERR $\gamma$ or Ad-ERR $\gamma$ S179A, and treated with forskolin $(10 \mu \mathrm{mol} / \mathrm{l})$ for $24 \mathrm{~h}$ or insulin for $18 \mathrm{~h} .{ }^{* * *} p<0.001 \mathrm{vs}$ forskolin and $* * * p<0.001$ vs Ad-ERR $\gamma$. Data are presented as means \pm SEM. The experiment was repeated on a minimum of three separate occasions

fasting blood glucose levels were significantly higher in Ad-ERR $\gamma$ wt or Ad-ERR $\gamma$ S179A-injected mice, compared 
with those of control mice (ESM Fig. 6). However, during insulin stimulation, blood glucose concentrations and hepatic gluconeogenic gene expression remained elevated in Ad-ERR $\gamma$ S179A-injected mice relative to Ad-ERR $\gamma$ wt-injected mice (Fig. 6a, b). Moreover, the occupancy of ERREs of Pckl promoter by ERR $\gamma$ S179A was unaffected by insulin (Fig. 6c). In addition, the phosphorylation and nuclear export of ERR $\gamma$ by insulin stimulation was exclusively detected in the liver of Ad-ERR $\gamma$ wt mice, but not in the liver of Ad-ERR $\gamma$ S179A (Fig. 6d, e). As expected, a phosphomimic mutant ERR $\gamma$ S179D exhibited cytoplasmic localisation (Fig. 6d). Finally, glucose excursions during the insulin tolerance test were higher in Ad-ERR $\gamma$ S179A-injected mice: these levels remained elevated in Ad-ERR $\gamma$ S179Ainjected mice compared with Ad-ERR $\gamma$ wt-injected mice for up to $2 \mathrm{~h}$ (Fig. $6 \mathrm{f}$ ). Taken together, these results suggest that the phosphorylation of ERR $\gamma$ at S179 enables insulin, at least in part, to control glucose homeostasis in mice.

\section{Discussion}

In this study, we identified orphan nuclear receptor ERR $\gamma$ as a novel substrate for PKB phosphorylation and demonstrated that ERR $\gamma$ is a major downstream mediator of insulin action on regulation of hepatic glucose metabolism (Fig. 6g). Interestingly, we found that the PKB phosphorylation site in ERR $\gamma$ is located near the second zinc finger of the DBD and its phosphorylation by insulin significantly decreases DNAbinding affinity of the receptor to the promoters of target genes. These findings are further supported by the result showing that the phospho-mimic mutant ERR $\gamma$ S179D
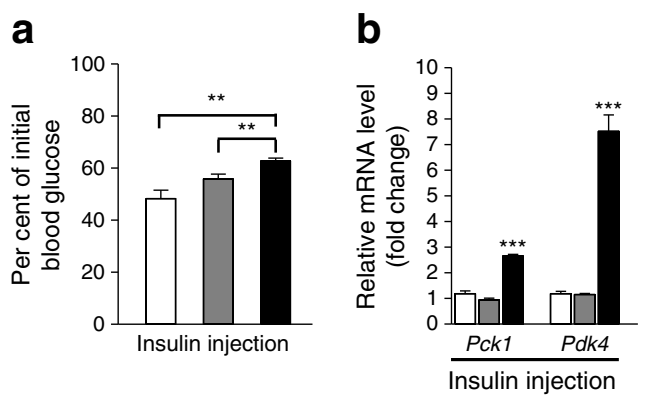

C

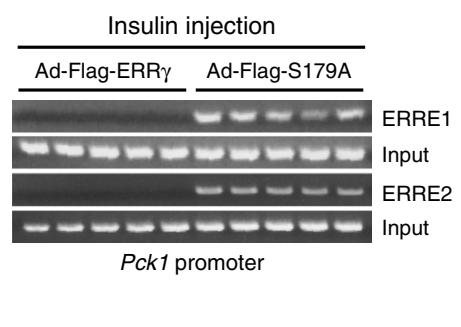

d

Insulin injection

e
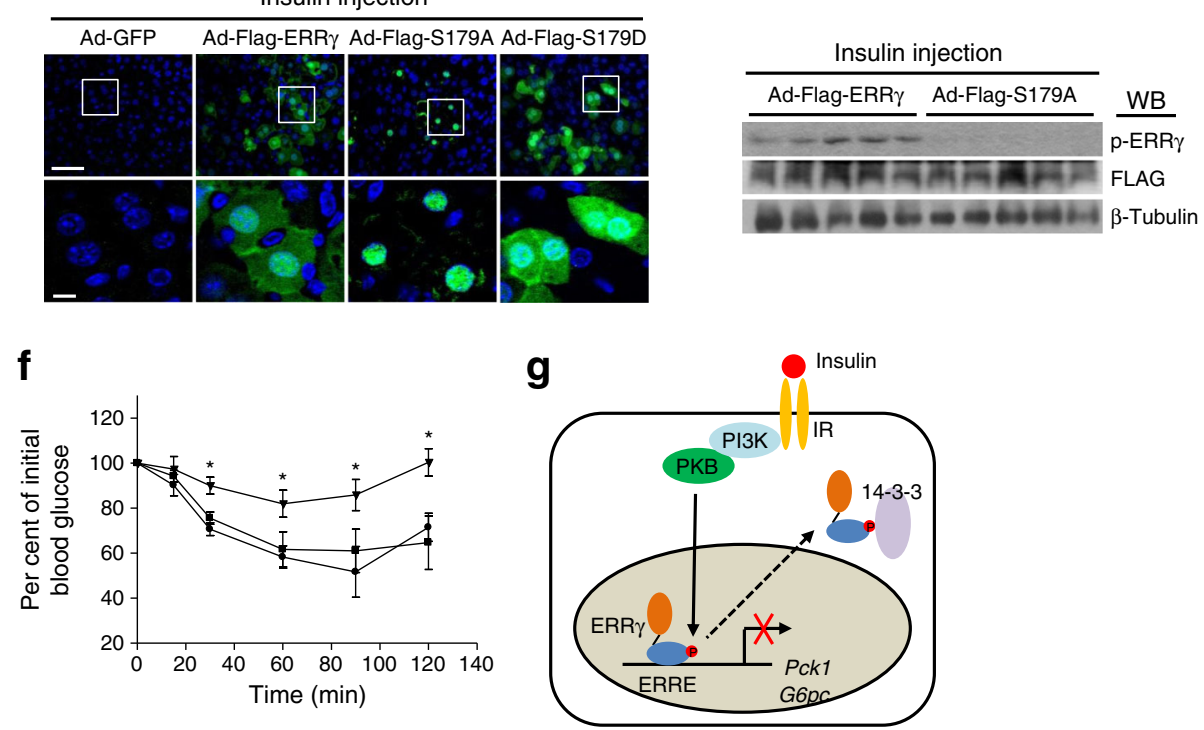

Fig. 6 Insulin inhibits hepatic gluconeogenesis through the phosphorylation of ERR $\gamma$ at S179 in mice. (a) Blood glucose levels. C57/BL6 mice ( $n=5$ per group) were infected with Ad-GFP (white bars), Ad-Flag-ERR $\gamma$ (grey bars) or Ad-Flag-ERR $\gamma$ S179A (black bars) and intraperitoneally injected with insulin $(1 \mathrm{U} / \mathrm{kg})$ for $1 \mathrm{~h}$ after $4 \mathrm{~h}$ fasting at day 7 of adenoviral infections; $*^{*} p<0.01$ as shown. (b) mRNA levels of Pckl and Pdk4 in mouse liver. ${ }^{* *} p<0.001$ vs Ad-GFP. (c) ChIP assay showing occupancy of ERR $\gamma$ and ERR $\gamma$ S179A on ERREs of $P c k 1$ promoter in mouse liver. (d) IHC showing subcellular localisation of ERR $\gamma$, ERR $\gamma$

S179A and ERR $\gamma$ S179D in mouse liver. IHC was performed using antiFLAG antibody. Scale bars, $50 \mu \mathrm{m}$ (top) and $10 \mu \mathrm{m}$ (bottom). (e) Western blot analysis showing phospho- and total ERR $\gamma$ protein levels in mouse liver. (f) Insulin tolerance test. C57/BL6 mice ( $n=5$ per group) infected with Ad-GFP (squares), Ad-Flag-ERR $\gamma$ (circles) or Ad-Flag-ERR $\gamma$ S179A (triangles) were intraperitoneally injected with $0.5 \mathrm{U} / \mathrm{kg}$ insulin after 4 h fasting. ${ }^{*} p<0.05$ vs Ad-GFP. (g) Schematic diagram of insulinmediated regulation of ERR $\gamma$ transcriptional activity. Data are presented as means \pm SEM 
displayed significantly lower basal transcriptional activity compared with ERR $\gamma$ wt. In the modelled ERR $\gamma$-DBD structure, S179 is located near the phosphate moieties of the bound dsDNA. Addition of a phosphate moiety on S179 provides a negative charge, which may detach the bound ERR $\gamma$-DBD from its cognate dsDNA due to negative charge repulsions present between the introduced phosphate group of negative charge on the S179 of ERR $\gamma$-DBD and the phosphate moieties of dsDNA. Similarly to these findings, it is reported that $E R R \alpha$ has four acetylation sites located in the DBD, and the acetylation changes DNA-binding and transcriptional activity [19]. Furthermore, the phosphorylation on the DBD of orphan nuclear receptor Nur77 also leads to a significant reduction of DNA-binding affinity of the receptor [20], suggesting that PTMs on DBDs of nuclear receptors could alter their transcriptional activity.

It is reported that, although ERRs possess similar functional domains and their transcriptional activity depends on interactions with similar coactivator or corepressor proteins, each ERR isoform sometimes exhibits different transcriptional outputs for the same target genes. For example, both ERR $\alpha$ and ERR $\gamma$ can positively regulate $P d k 4$ expression and they have the potential to control mitochondrial programmes implicated in oxidative phosphorylation, while SHP and Lipin1 expression is regulated by ERR $\gamma$ but not by ERR $\alpha$ or $\operatorname{ERR} \beta[12,17$, $21,22]$. Moreover, PGC-1 $\alpha$-mediated induction of Pckl expression was mediated by ERR $\gamma$, but inhibited by ERR $\alpha$ $[10,23]$. In addition to the complexity of their function in the regulation of target genes, the regulation of PTMs for each ERR is also complicated. Indeed, both ERR $\alpha$ and ERR $\gamma$ undergo PTMs such as sumoylation of the amino-terminal domain and acetylation of the DBD [19, 24], whereas ERR $\gamma$, but not $\mathrm{ERR} \alpha$, is phosphorylated by the insulin signalling pathway in this study. These results suggest that PTM of ERRs could be a key mechanism leading to alteration of their transcriptional outputs. Therefore, a specific physiological cue causing PTMs of each ERR needs to be determined.

Hormonal regulation of hepatic glucose metabolism during fasting and feeding is important for whole-body glucose homeostasis. Although the pancreatic hormone insulin is known to inhibit HGP mainly through nuclear export of FOXO1 by PKB-mediated phosphorylation, several pieces of evidence indicated that loss of Foxol function does not completely abolish the insulin regulation of hepatic gluconeogenesis [25-27], suggesting that additional factors are at play. Here, we demonstrated that insulin-dependent phosphorylation of ERR $\gamma$, leading to its nuclear export, is a major contributor to the inhibitory action of insulin on HGP. Moreover, a phosphorylation-deficient mutant, ERR $\gamma$ S179A, is resistant to insulin action on hepatic gluconeogenesis. We also showed that elevated ERR $\gamma$ phosphorylation in WT mice during feeding was not found in LIRKO or $P \mathrm{~kb}^{-/-}$mice, demonstrating that ERR $\gamma$ is a major transcriptional mediator for maintaining the inhibitory action of insulin on hepatic gluconeogenesis. These results suggest that a defect of insulin action on ERR $\gamma$ transcriptional activity may contribute to hepatic insulin resistance through an inability to phosphorylate ERR $\gamma$. In addition, generation of ERR $\gamma$ S179A knock-in mice would provide further insights into the role of ERR $\gamma$ in insulin action on the hepatic gluconeogenic programme. In conclusion, we propose that the inhibition of hepatic ERR $\gamma$ activity would suppress the additional increase of blood glucose by the abnormal induction of hepatic gluconeogenesis in type 2 diabetes.

Acknowledgements We would like to thank D. D. Moore (Baylor College of Medicine, Houston, TX, USA) and S.-Y. Choi (Chonnam National University Medical School, Gwangju, Republic of Korea) for critical reading and helpful discussions of the manuscript.

Funding This work was supported by a National Creative Research Initiatives Grant (20110018305) through the National Research Foundation of Korea (NRF) funded by the Korean government (Ministry of Science, ICT \& Future Planning) to H-SC; DK094162 (to SBB); and a grant of the Korea Health Technology R\&D Project (A111345) funded by the Korean government (Ministry of Health \& Welfare) to I-KL.

Duality of interest The authors declare that there is no duality of interest associated with this manuscript.

Contribution statement D-KK, Y-HK, DH, YW, K-JY, DR, KSK and $\mathrm{E}-\mathrm{KY}$ performed the experiments or analysis and interpretation of data. J-SK, S-HK, I-KL, H-ZC, JP, C-HL, SBB and BAH provided materials for experiments and contributed to conception and design, acquisition of data, or analysis and interpretation of data. D-KK and H-SC analysed the data and wrote the paper. All authors contributed to the discussion and revised the article and all approved the final versions of the manuscript. $\mathrm{H}-\mathrm{SC}$ is responsible for the integrity of the work as a whole.

\section{References}

1. Saltiel AR (2001) New perspectives into the molecular pathogenesis and treatment of type 2 diabetes. Cell 104:517-529

2. Pilkis SJ, Granner DK (1992) Molecular physiology of the regulation of hepatic gluconeogenesis and glycolysis. Annu Rev Physiol 54: 885-909

3. Yabaluri N, Bashyam MD (2010) Hormonal regulation of gluconeogenic gene transcription in the liver. J Biosci 35:473-484

4. Kim DH, Perdomo G, Zhang T et al (2011) FoxO6 integrates insulin signaling with gluconeogenesis in the liver. Diabetes 60:2763-2774

5. Dentin R, Liu Y, Koo S-H et al (2007) Insulin modulates gluconeogenesis by inhibition of the coactivator TORC2. Nature 449:366-369

6. Li X, Monks B, Ge Q, Birnbaum MJ (2007) Akt/PKB regulates hepatic metabolism by directly inhibiting PGC-1alpha transcription coactivator. Nature 447:1012-1016

7. Tremblay AM, Giguere V (2007) The NR3B subgroup: an ovERRview. Nucl Recept Signal 5:e009

8. Kim DK, Kim YH, Jang HH et al (2013) Estrogen-related receptor gamma controls hepatic $\mathrm{CB} 1$ receptor-mediated CYP2E1 expression and oxidative liver injury by alcohol. Gut 62:1044-1054

9. Kim DK, Jeong JH, Lee JM et al (2014) Inverse agonist of estrogenrelated receptor gamma controls Salmonella typhimurium infection by modulating host iron homeostasis. Nat Med 20:419-424 
10. Kim DK, Ryu D, Koh M et al (2012) Orphan nuclear receptor estrogen-related receptor gamma (ERRgamma) is key regulator of hepatic gluconeogenesis. J Biol Chem 287:21628-21639

11. Kim DK, Gang GT, Ryu D et al (2013) Inverse agonist of nuclear receptor ERRgamma mediates antidiabetic effect through inhibition of hepatic gluconeogenesis. Diabetes 62:3093-3102

12. Kim DK, Kim JR, Koh M et al (2011) Estrogen-related receptor gamma (ERRgamma) is a novel transcriptional regulator of phosphatidic acid phosphatase, LIPIN1, and inhibits hepatic insulin signaling. J Biol Chem 286:38035-38042

13. Fisher SJ, Kahn CR (2003) Insulin signaling is required for insulin's direct and indirect action on hepatic glucose production. J Clin Invest 111:463-468

14. Dummler B, Tschopp O, Hynx D, Yang ZZ, Dirnhofer S, Hemmings BA (2006) Life with a single isoform of Akt: mice lacking Akt2 and Akt3 are viable but display impaired glucose homeostasis and growth deficiencies. Mol Cell Biol 26:8042-8051

15. Woo HA, Kang SW, Kim HK, Yang KS, Chae HZ, Rhee SG (2003) Reversible oxidation of the active site cysteine of peroxiredoxins to cysteine sulfinic acid. Immunoblot detection with antibodies specific for the hyperoxidized cysteine-containing sequence. J Biol Chem 278:47361-47364

16. Yang KJ, Shin S, Piao L et al (2008) Regulation of 3-phosphoinositidedependent protein kinase-1 (PDK1) by Src involves tyrosine phosphorylation of PDK1 and Src homology 2 domain binding. J Biol Chem 283:1480-1491

17. Sanyal S, Kim JY, Kim HJ et al (2002) Differential regulation of the orphan nuclear receptor small heterodimer partner (SHP) gene promoter by orphan nuclear receptor ERR isoforms. J Biol Chem 277: $1739-1748$
18. Tzivion G, Shen YH, Zhu J (2001) 14-3-3 proteins; bringing new definitions to scaffolding. Oncogene 20:6331-6338

19. Tremblay AM, Wilson BJ, Yang XJ, Giguere V (2008) Phosphorylation-dependent sumoylation regulates estrogen-related receptor-alpha and -gamma transcriptional activity through a synergy control motif. Mol Endocrinol 22:570-584

20. Hirata Y, Kiuchi K, Chen HC, Milbrandt J, Guroff G (1993) The phosphorylation and DNA binding of the DNA-binding domain of the orphan nuclear receptor NGFI-B. J Biol Chem 268:24808-24812

21. Zhang Y, Ma K, Sadana P et al (2006) Estrogen-related receptors stimulate pyruvate dehydrogenase kinase isoform 4 gene expression. J Biol Chem 281:39897-39906

22. Dufour CR, Wilson BJ, Huss JM et al (2007) Genome-wide orchestration of cardiac functions by the orphan nuclear receptors ERRalpha and gamma. Cell Metab 5:345-356

23. Herzog B, Cardenas J, Hall RK et al (2006) Estrogen-related receptor alpha is a repressor of phosphoenolpyruvate carboxykinase gene transcription. J Biol Chem 281:99-106

24. Wilson BJ, Tremblay AM, Deblois G, Sylvain-Drolet G, Giguere V (2010) An acetylation switch modulates the transcriptional activity of estrogen-related receptor alpha. Mol Endocrinol 24:1349-1358

25. Matsumoto M, Pocai A, Rossetti L, Depinho RA, Accili D (2007) Impaired regulation of hepatic glucose production in mice lacking the forkhead transcription factor Foxo1 in liver. Cell Metab 6:208-216

26. Haeusler RA, Kaestner KH, Accili D (2010) FoxOs function synergistically to promote glucose production. J Biol Chem 285:3524535248

27. Lu M, Wan M, Leavens KF et al (2012) Insulin regulates liver metabolism in vivo in the absence of hepatic Akt and Foxo1. Nat Med 18:388-395 